\title{
Sachregister zu Band 91
}

Die lett gedruckten Zahlen bedeuten Eigenarbeiten. Bb. = Buchbesprechungen.

Ablatio retinae 74ff $\cdot$, 102, 242, 257. 258, 307

ohne Foramen 224

Behandlung der - 366 Adaptation 201 Adipositas, Keratokonus bei ungewöhnlicher - -238 Akiyami 54, 63 Albinismus, Vererbung des

296 Alkoholbehandlung des Blepha-

rospasmus infolge von post-

encephalitischem Parkinsonis-

mus 230 Allergiebedingte Lídödeme

262 Alterserscheinungen am Auge

$3^{\circ} 9$ Amaurotische Idiotie, familiäre

247. 305 Amylnitrit, Einatmungen von

bei Erkrankungen des Augen-

hintergrundes 357 Anatomie 112 (Bericht) Anenzephalie 97 Angiom der Chorioidea 257

- der Orbita 257Angiomatosis retinae 83, 230,

307 Angioneurose, chronisches inter-

mittierendes Hornhautödem als

Ausdruck einer - -278 Angiopathia retinae trauma-

tica 58, 241 Angiospasmus der Netzhaut-

gefäße 69 Aniridie, erbliche 98, 231, 294 Anisometropie 300 Anophthalmus 96. Area des

Gehirns, angioarchi-

tektonischer Bau der - 258 Argidal 353 Arsenvergiftung, Augenschädi-

gung bei -58 Arteria centralis retinae, Ver-

schluß der -69 Arteria hyaloidea persistens

100 Arteriosklerose, Retinopathie

bei - 66 Atophanyl mit Cylotropin bei

sympathischer Ophthalmie 356 Atropin, retrobulbäre Injektionen

von -358

Augenfarbe, Vererbung der -

295 Augenmuskeln, Anatomie der -

122

- $\quad$ erbliche Erkrankungen der äu-ßeren —-ロ 293

Augenrollung, Messung der -

260 Autovakzinebehandlung 353 Avitaminosen, die B-Av. des

Auges 227

Bindehaut s. Conjunctiva

Blepharo-Chalasis 238

Blepharophimosis, Stamm-baum von - 293 
Blepharospasmus infolge von postenzephalitischem Parkinso-nismus, Alkoholbehandlung des 230

Blepharospasmusbrille 289

Blindheit und Eugenik 310

Blutgefäßgeschwülste in Retina und Zentralnervensystem 126

Blutkrankheiten, Augenhinter-grund bei - 70

Blutverlust, Netzhautverände-rungen nach -69

Botulismus, Sehstörungen bei -60

Brille 227

- Geschichte der - noBrillensteg, fehlerhafter als Ur-

sache von Krebs 185

Chininvergiftung, Sehstörungen bei -58 Chorioidea 53 (Bericht)

Angiome der $\mathbf{a}-\cdot 257$

nekrotisches Sarkom der - 220 Chorioretinitis centralis se-

rosa 62 Conjunctiva, Anatomie der -

"3

- Wasserstoffionenkonzentrationdes Sekrets der -- 324

Karzinom der - 255 Cornea, Anatomie der - 114

Entwicklung der - -94

Architektur der -258

-- erbliche Affektionen der - 300

- $\quad$ symmetrische Entartung der $-\mathbf{m} 253$

$25^{*}$

380 Sachregister zu Band 91.

Cornea, zyklisch auftretendes

Ödem der - -228 - chronisches intermittierendes

Ödem der als Ausdruck einer

Angioneurose 278 - Infiltration der - - und Ulzera-

tion 235 - - Überpflanzung von - 230, 362 Corneatrübung, angeborene fa-

miliäre - 326 - subepitheliale - bei Pagetscher

Krankheit 378 - bandförmige - - bei der Polyarthritis leucocytica und Гym-

phocytica 325 Cyclodiathermiestichelung

221 Cylotropin mit Atophanyl bei

sympathischer Ophthalmie 356 Cysticercus 63

Dakryostenose, angeborene 102 Dakryozystoblennorrhoe nach

Exstirpation des Tränensackes

217 Dakryozystorhinostomie 367 - Modifikation der - 140 Dermoidzysten 104 Diabetes

mellitus, hintere Scha-

lenkatarakt und Linsenmyopie

bei $\mathbf{a}-218$

- $\quad$ Retinopathie bei - 67Diathermie 370

- diaphanoskopische - bei Ablatio retinae 80

- des Corpus ciliare zur intra-okularen Druckherabsetzung 221

Doppeltsehen bei Siebbeinkarzi-nom 236

Doryl 356

Druck, intraokularer $63 \mathrm{ft}$. 
-- Herabsetzung des - mittels üiathermieschädigung des Corpus ciliare 221

Ductus nasolacrimalis, künst-liche Wiederherstellung des - 50

Dyslipoidosen 305

Dysostosis craniofacialis 104

Eidetik 208 Eigenblutinjektionen in die

Vorderkammer bei Keratitis par-

enchymatosa 355 Einschlußkörperchen 113 Ektopia lentis 101

Vererbung der

294

Elektrischer Strom, Augen-

schädigungen durch den $\mathbf{m}-58$ Elektrokoagulation bei Ablatio

retinae 79 Endophthalmitis septica 59

Endothelioma psammosum

257 Entwicklungsgeschichte des

Auges 93 (Bericht) Epikanthus, erblicher 102 -- angeborener - mit Ptosis 292 Epileptischer Anf all, Netzhaut-

befunde vor dem - 68 Epitheldystrophie, juvenile 185 Erythema nodosum, Chorioi-

ditis bei - 55 Erythromelalgie, Netzhautver-

änderungen bei - 70 Eugenik und Blindheit 310. Exophthalmus infolge eines ent-

zündlichen Ödems der Orbita

237

Far ben, Wirkung von -208

Farbenblindheit 20

Farbensinn 194

- Prüfung des $\cdot-242$

- angeborene Störungen des -

304 Feuerlamelle, beiderseitige -

bei Glasbläserstar 214 Fib rose, massive - der Netzhaut

261 Fieber, künstliches - bei Augen-

syphilis 327 Fleckfieber, Augenstörungen bei

- 60 Flimmerskotom 327 Fokalinfektion 60 Formwahrnehmungen 209 Fremdkörperverletzung

235 Frühjahrskatarrh und Limbus-

wucherungen 254 Fusionsstörungen nach Gehirn-erschütterungen 377

Gefäßkreuzungen am Augen-hintergrund 322

Gehirnausbildung und Refrak-tion 299

Gehirnerschütterung, Fusionsstörungen nach घ- 377

Gehirnsklerose, tuberose 83

Gehirnverletzung, Erkennung der traumatischen - -378

Geschichte der Augenheilkunde 108

Geschwülste 116, 255ff.

- Vererbung von $\cdot-297$

Geschwulstwachstum und Un-fallwirkung 125

Gesichtsfeld 200

Gestaltswahrnehmungen 209

Glasbläserstar mit beiderseitiger Feuerlamelle 214

Glaskörper, Anatomie des - 119

Sachregister zu Band 91.

381 
Glaskörperblutungen 218 - rezidivieren.de 238 Glaukom, Vererbung des -ロ 296 Glaukomatöse Exkavation,

wechselnde 229 Gleichgewichtsapparat, Unter-

suchung des $\mathbf{-}$ - bei Begutach-

tung von Kopfverletzvmgen 374 Gliom des Sehnerven und der

Netzhaut 84, 257 - der Netzhaut durch Röntgen-

strahlen geheilt 348 Grippe, Netzhautblutungen bei

60 Größenwahrnehmungen 209 Großflächenpolarisatoren 248 Gum ma der Bulbusbindehaut bei

Lues congenita 273

Hämangiom des Oberlides, durch

Radium geheilt 247 Hemeralopie, Augenhintergrund

bei - 71 Herbstfieber 54, 63 Herpes corneae 258 Heterochromie 99, 296 Heufieber,

Behandlung des -354 Hochdruck, Retinopathie bei -

66 Hornhaut s. Cornea Hydrophthalmus congenitus

und Naevus flammeus 95 Hyperpigmentierung des Auges

85

Hypertelorismus 104

Hypopyoniritis, rezidivierende 129, 320

Hypovitaminose und Adaptation 201

Ignipunktur bei Ablatio retinae

77 Iontophorese 369 Iridozyklitis, rezidivierende 230 Iris, erbliche Affektionen der -

302

angeborene Anomalien eines Ge-fäßes der $\mathbf{-}-322$

Durchblutung der - bei Throm-bopenie 229

Iriszyste 215

Iritis, rezidivierende Hypopyon-I. 129, 320

- $\quad$ tuberkulöse - nach rheumati-scher 218

Joddämpfe, therapeutische An-wendung der - 353

Kala-Azar, Augensymptome bei

- 63 Kapillariitis der Netzhautmitte

72

Karzinose des Auges 256 Katarakt, angeborene $100 \bullet-$ Vererbung der $\cdot-303$

$\mathrm{K}$. filiformis 338

Glasbläserstar mit beiderseitiger Feuerlamelle 214

Röntgenstar auf dem nicht-bestrahlten Auge 219

medikamentöse Therapie der senilen $\cdot-231$

Kataraktextraktion 364

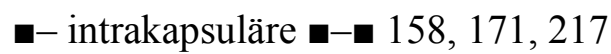

Katholyse, Blutungsgefahren bei

der - 226 Keratitis parenchymatosa 236 - und Lues congenita der 2. Generation 183, $264-$

Eigenblutinjektionen in die Vor-

derkammer bei --355 - Hornhauttransplantation bei -

230 Keratitis superficialis, Poilen-allergie als Ursache einer - 343 Keratokonus 220, 301

- $\quad$ bei ungewöhnlicher Adipositas238

Kernschwund, infantiler 293 
Kinematographie in der medi-zinischen Forschung 248

Kleinhirnataxie, Augenhintergrund bei hereditärer $\mathbf{-}-72$

Knochenbildung in der Ader-haut 53

Kollargolsalbe zur Prophylaxe gegen Ulcus serpens 265

Kolobome 97

Vererbung der $\mathbf{-}-294$

des gelben Fleckes 84

der Linse 101

der Lider 102 Konsanguinität 309 Kontax 248 Kopfverletzungen, Untersu-

chung des Gleichgewichtsappa-rates bei Begutachtung von -

374 Krebs durch fehlerhaften Brillen-steg verursacht 185

- beiXeroderma pigmentosum 255

- $\quad$ metastatischer - im Ziliarkör-per 329

L-aurence-Biedlsches Syn-drom 71, 72, 229, 306

Lehrfilm 247

Lentikonus posterior 101

Leontiasis ossea 251

Leontiasisartige Verdickung an Stirn und Schläfe 236

Lepra, Augenbefunde bei - 55

Lichtsinn 187

Lid, Milzbrand des - 233

382

Sachregi ${ }^{7} / 8$ ter zu Band 91.

Lidabszeß 235, 236

Lidkankroide 240

- - Behandlung der - mit Thorium

X 251 Lidödeme, allergiebedingte 262 Lidplastik 360, 362 Limbuswucherungen und Frühjahrskatarrh 254 Linse, Anatomie der - 118 -- erbliche Affektionen der - 303

- $\quad$ Randwülste der normalen lu-xierten - 253

Linsenkapselabschilferung

mit Glaukom 220 Lipämie der Netzhaut 67 Lupus erythematosus, Ader-

hautherde bei - 55 Lymphogranuloma inguinale,

Netzhautveränderungen bei -

60

Macula lutea, Fehlen der -bei

2 Schwestern 231 - - Heredodegeneration der $\mathbf{m}-72$,

305 - Kolobom der - 84, 97 Malaria, Chorioiditis bei - 55 - Netzhautblutungen bei - 63

Maltafieber, Augenstörungen bei

- 60 Megalokornea 98 Melanoblastome der Haut mit

Pigmentnaevus 256 Melanom, malignes - der Ader-

haut 56 Melanosarkom der Bulbusbinde-

haut $221 \bullet-$ am Makularande 241 Melanosis des Augapfels 99 Menformon 357 Methylalkohol,

Netzhautschädi-

gung durch - 58 Mikrokornea 98 Mikrophthalmus 96 Mikuliczsche Tumoren 237 Milzbrand des Augenlids 233 Mißbildungen 95 (Bericht) Mongolismus 105 Myopie, Vererbung der •-298 
- $\quad$ Böhms Vererbungsgesetze der-183

- Aderhautdegeneration bei-56 Myxosarkom 256

Naevus flammeusund Glaukom 296

- $\quad$ und Hydrophthalmus congeni-tus 95

Naevus pigmentosus, Melanoblastome der Haut bei $\mathbf{-}-256$

Nagelfalzblutungen, kapilläre 264

Naturheilkunde 260, 352 Nervus opticus, Anatomie des - -121

erbliche Affektionen des -308

Neuritis des bei - Wurzel-spitzengranulom 322

Nervus opticus-atrophie 240 - Behandlung der tabischen

358 Netzhaut s. Retina Neurinom des Sehnerven 220 Neurofibrillitis 61 Neuroretinitis septica 59

Nierenleiden, Retinopathie bei

- $\quad$ 67Nitrobenzolvergiftung, Augen-

veränderungen bei - 58 Nystagmus, Vererbung des - 294 - bei der totalen angeborenen

Farbenblindheit 20

- Prognose für die Sehschärfe beiangeborenem - 104

- - N. retractorius 249

Oguchische Krankheit 70 Oktinum 356

Onchocercus volvulus 56 Ophthalmia metastatica59,236 Ophthalmia sympathica 117 - nach

Aderhauttumor 55 - Netzhautveränderungen bei -62

- $\quad$ Atophanyl mit Cylotropin bei -356

Ophthalmoskop der Lausanner

Klinik 224 Optik, physiologische 187 (Bericht)

- $\quad$ psychologische 208 (Bericht)Orbitalsarkom 256Orbitaltumor vaskulärer Art

230Osteodermatopathie 231Osteopsathyrosis und blaue

Sklera 233 Ozonbehandlung, subkonjunk-tivale 227

Pagetsche Krankheit, symme-trische subepitheliale Hornhaut-trübung bei - 378

Panophthalmie 234

Pantocain 245

Parkinsonismus, Alkoholbe-handlung des Blepharospasmus infolge von postenzephaliti-schem

- 230

Pathologische Anatomie 112 (Bericht)

Pellagra, Aderhautblutung bei

- 56Periphlebitis retinae 61Phlebitis juvenilis retinae.

Riesenzellen bei - 221

Sachregister zu Band 91.

383

Photographie in der medizini-

schen Forschung 248 Physikalische Behandlung 369 Physiologische Optik 187 (Ber.)

Pigmentdegeneration der Netz-

haut 71 Pigmentierung, Vererbung der

- 295Pigmentstreifenerkrankung,

angioide - des Fundus 73, 307 Plasmocid, Sehstörungen durch

- $\quad$ 59Polarisationsfilter 249Pollenallergie als Ursache einer

Keratitis superficialis 343 
Polyarthritis acuta, Chorioi-ditis bei - 54

Polyarthritis leucocytica und lymphocytica, bandförmige Trübungen der Cornea bei - 325

Polykorie 99

Preglsche Jodlösung, Sehstörungen durch - 59

Projektionsperimeter 255

Prostigmin 355

Pseudotumor, Histogenese des senilen submakulären - -223

Pseudoxanthoma elasticum und angioide Streifen 307

Psychologische Optik 208 (Be-richt)

Ptosis, angeborene 103, 292, 293

Pupille, erbliche Affektionen der

$-\quad 302$

- Dauerlähmung der linken - 241Pupillenabstand bei Japanern

108 Pupillotonieinder Begutachtung müitärischer Dienstfähigkeit 251 Purkinjesches Phänomen 192 Pyelonephritis, Panophthalmie bei -53

Radiumbehandlung 373

-- Heilung eines Hämangioms des

Oberlides durch $\mathbf{\square}-247$ Radiumemanation, Lokalbe-

handlung von Augenleiden mit

- $\quad$ 124Raumsinn 203Raumwahrnehmungen 209RaynaudscheKrankheit, Netz-

hautveränderungen bei - -70 Recklinghausensche Krank-heit, Augenbefund bei - $-\mathbf{\square} 84$

- $\quad$ erbliche 292R edoxon 355

Refraktionsanomalien, Vererbung der $\cdot-298$

Resistenzminderwertigkeit, organgebundene hereditäre - 219

Retina 58 (Bericht)

- pathologische Anatomie der - 120

- $\quad$ erbliche Affektionen der - 304Blutgefäßgeschwülste in der

- -126

- $\quad$ massive Fibrose der $\mathbf{m}-261$ - Zysten der 244Retinafalte, angeborene 84,

102Retinagefäße, Messungen des

Durchmessers der - 249

- $\quad$ Anomalien der - 85Retinariß, Lokalisation des -

während der Operation 225

- $\quad$ Heilung eines - ohne Amotio225

Retinitis exsudativa Coats 82 ,

241 Retinitis pigmentosa 306 - mit Keratokonus congenitus 220 Röntgenbehandlung 371

- $\quad$ eines Netzhautglioms 348Röntgenstar auf dem nichtbe-

strahlten Auge 219

Salpetersäureverätzung 239 Schädel, Anomalien des - 309 Schlaf krankheit, Chorioiditis bei - 56 Schleimhauttransplantation

nach Denig-Neumann 224 Schwangerschaft, Retinopathie

bei - 68 Schwefelsäureverätzung 238,

239 Sehbahn, Anatomie der - 121 Sehnerv s. Nervus opticus. Sehschärfe 203 Sehzentrum, Architektur des

kortikalen - 327 Siebbeinkrebs 256 
Doppeltsehen bei $\cdot-236$ Sklera, Anatomie der - 114 erbliche Affektionen der - 300 •- blaue 98, 233 Skleralzyste 242 Spondylarthritis ankylopoetica, Iritis bei - 54 Sprengstoffexplosion 239 Stereoskopie des Röntgenbildes

205 Strahlenschädigung der Netz-

haut 58 Sudankrankheit 56 Symblepharon posterius superius bei Trachom 219 Synästhesie 208 Syntropan 356 Syphilis, Aderhauterkrankungen bei -54

- $\quad$ Fieberbehandlung der Augen-S.327

384

Namenregister zu Band 91.

Syphilis, gummöser Prozeß am oberen Orbitalrande 236

Syphilis congenita der zweiten Generation und Keratitis paren-chymatosa 183, 264

- $\quad$ Gumma der Bulbusbindehautbei - 273

Tenotomia filiformis 228 Therapie, medikamentöse 352

(Bericht) •- operative 360 (Bericht)

- $\quad$ physikalische 369 (Bericht)Thorium X in der Behandlung

der Lidkankroide 251 Tiefensehen 203 Thrombopenie, Durchblutung

der Iris bei - 229 Touraine-Solente-Golésches

Syndrom 231, 292 Trachocid 216, 354 Trachom 113, 116 -- Symblepharon posterius supe-

rius bei - 219 - Behandlung des 354 Tränenflüssigkeit, Wasserstoff-

ionenkonzentration der $\mathbf{\square}-324$ Tränenorgane, Anatomie der

112 Trichiasis, Operation der - 361,

362 Tuberkelbazillämie 262 Tuberkulose der Haut und Binde-

haut 321 Tuberkulose des Auges 54, 60•-des Augenhintergrundes 218

Tularämie 1 Turmschädel 104, 309

Ulcus serpens, atypisches $235 \bullet-$ Kollargolsalbe zur Prophylaxe

gegen 265 Ultrakurzwellen 371 Unfallbegutachtung, patholo-

gische Anatomie im Dienste der

-263 Unf allwirkung und bösartiges

Geschwulstwachstum 125 Unguentolan 355

Vererbung und Auge 292 (Bericht) Vitaminbehandlung 354

Wasserstoffionenkonzentra-tion der Tränenflüssigkeit und des Conjunctivalsekrets 324

Xanthomatosen 72 Xeroderma pigmentosum und

Pigmentdegeneration 72 - Karzinom bei - 255

Zahnwurzel, Granulom der - und Neuritis optica 322

Zeitlupenvorführung 248

Zentralnervensystem, Blut-gefäßgeschwülste am - 126

Ziliarkörper, metastatisches Karzinom im $<-329$

- Sarkom des a- 214

Zonulafasern, Operationstechnik zur Zerreißung der @- 171

Zwillingsuntersuchung 309

Zyklopie 95

Namenregister zu Band 91. 
Abdulajew 362 Abramowicz 74 Adachi 73 Adda 329 Addario 57 Adrogué 71, 306 Agadjanian 208 Aglialoro 68 Agnello 66 Akiya 58 Alaerts 207 Albrich 116 Alcalá Lopez 10 Amemiya 63 Ames 206 Amsler 77, 222,

2. 225, 229 Anastasi 209 